\title{
Clinical effects and treatment of envenoming by Hoplocephalus spp. snakes in Australia: Australian Snakebite Project (ASP-12)
}

\author{
G.K. Isbister ${ }^{\mathrm{a}, \mathrm{b}, *}$, J. White ${ }^{\mathrm{c}}$, B.J. Currie ${ }^{\mathrm{d}}$, M.A. O'Leary ${ }^{\mathrm{b}}$, S.G.A. Brown ${ }^{\mathrm{e}, \mathrm{f}}$, for the ASP Investigators \\ a Discipline of Clinical Pharmacology, University of Newcastle, c/o Calvary Mater Newcastle, Edith St, Waratah NSW 2298, Australia \\ ${ }^{\mathrm{b}}$ Department of Clinical Toxicology and Pharmacology, Calvary Mater Newcastle, NSW, Australia \\ ${ }^{\mathrm{c}}$ Department of Toxinology, Women's and Children's Hospital, Adelaide, SA, Australia \\ ${ }^{\mathrm{d}}$ Menzies School of Health Research and Northern Territory Clinical School, Darwin, Northern Territory, Australia \\ e Centre for Clinical Research in Emergency Medicine, Western Australian Institute for Medical Research, Royal Perth Hospital, Australia \\ ${ }^{\mathrm{f}}$ University of Western Australia, WA, Australia
}

\section{A R T I C L E I N F O}

\section{Article history:}

Received 20 June 2011

Received in revised form 15 September 2011

Accepted 22 September 2011

Available online 28 September 2011

\section{Keywords:}

Hoplocephalus

Snake venom

Antivenom

Envenoming

Stephens banded snake

\begin{abstract}
A B S T R A C T
There is limited information on envenoming by snakes of the genus Hoplocephalus from Eastern Australia. We investigated the clinical and laboratory features of patients with definite Hoplocephalus spp. bites including antivenom treatment, recruited to the Australian Snakebite Project. There were 15 definite Hoplocephalus spp. bites based on expert identification including eight by Hoplocephalus stephensi (Stephen's banded snakes), four by Hoplocephalus bungaroides (broad-headed snake) and three by $H$. bitorquatus (paleheaded snake). Envenoming occurred in 13 patients and was similar for the three species with venom induced consumption coagulopathy (VICC) in all envenomings. Seven patients had an INR $>12$ and partial VICC, with only incomplete fibrinogen consumption, occurred in three patients. Systemic symptoms occurred in eight patients. Myotoxicity and neurotoxicity did not occur. $H$. stephensi venom was detected in all three $H$. stephensi envenomings (1.1, 44 and $81 \mathrm{ng} / \mathrm{mL}$ ) for whom pre-antivenom blood samples were available, and not detected in one without envenoming. In two cases with post-antivenom blood samples, venom was not detected after tiger snake antivenom (TSAV) was given. In vitro binding studies demonstrated that TSAV concentrations of $50 \mathrm{mU} / \mathrm{mL}$ are sufficient to bind the majority of free $H$. stephensi venom components at concentrations above those detected in envenomed patients $(100 \mathrm{ng} / \mathrm{mL})$. Eleven patients received antivenom, median dose 2 vials (Range: 1 to 5 vials), which was TSAV in all but one case, where polyvalent antivenom was used. Immediate hypersensitivity reactions occurred in six cases including one case of anaphylaxis. Envenoming by Hoplocephalus spp. causes VICC and systemic symptoms, making it clinically similar to brown snake (Pseudonaja spp.) envenoming. Based on in vitro studies reported here, patients may be treated with one vial of TSAV, although one vial of brown snake antivenom may also be sufficient.
\end{abstract}

(c) 2011 Elsevier Ltd. All rights reserved.

\section{Introduction}

The genus Hoplocephalus includes three different species of snakes that are relatively rare and confined to

\footnotetext{
* Corresponding author. Discipline of Clinical Pharmacology, University of Newcastle, c/o Calvary Mater Newcastle, Edith St, Waratah NSW 2298, Australia. Tel.: +61 24921 1211; fax: +61 249211870 .

E-mail address: geoff.isbister@gmail.com (G.K. Isbister).
}

small geographical regions in Eastern Australia. Stephen's banded snake (Hoplocephalus stephensi) is the most common and distributed throughout northern coastal New South Wales (NSW) (Figs. 1 and 2). The broad headed snake (Hoplocephalus bungaroides) is confined to a smaller region in central coastal NSW and the pale headed snake $(H$. bitorquatus) to a larger but relatively unpopulated region of south western Queensland (Figs. 1 and 2). All species are relatively commonly kept by herpetologists and private 


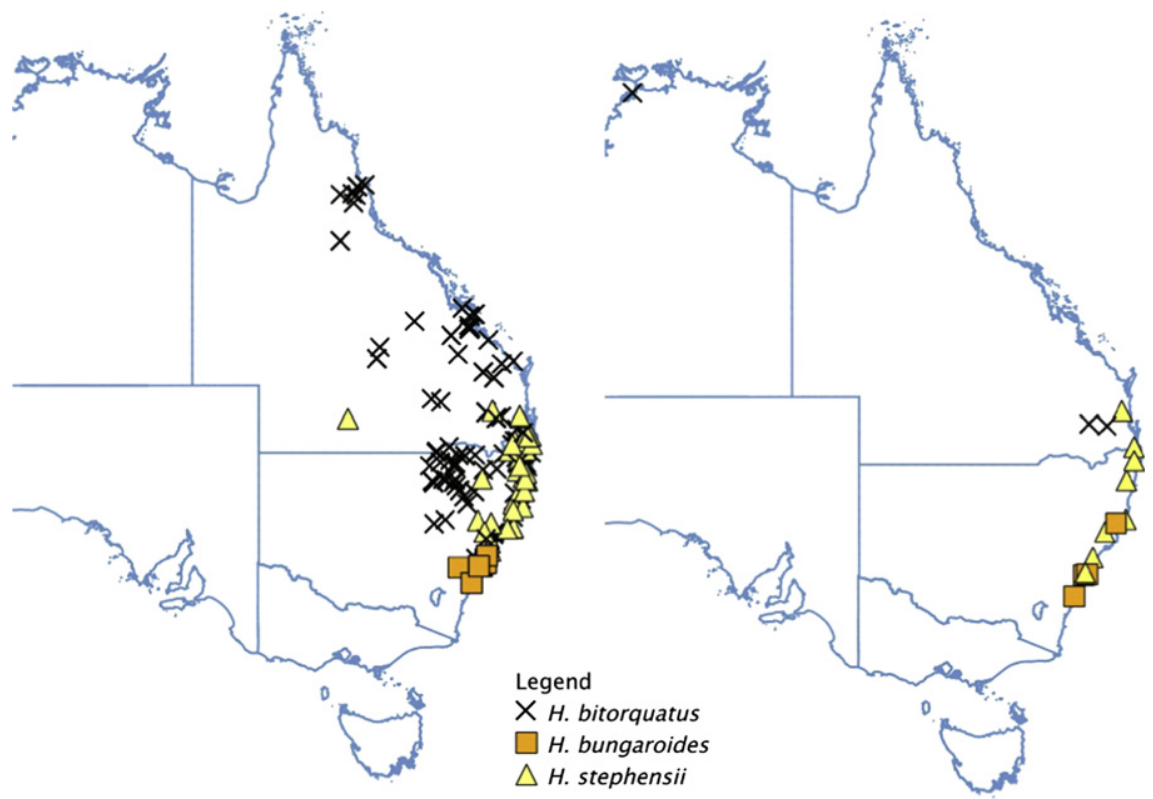

Fig. 1. Distribution maps of specimens of H. stephensi ( $\triangle$ ), H. bungaroides ( $\square$ )and H. bitorquatus $(\times)$ from museum data (left panel) compared to locations of bites by the three species (right panel).

collectors and most previously reported bites by Hoplocephalus snakes have been in these people (Flecker, 1952; Comadira and Green, 1995; Fry et al., 2000; Sutherland and Tibballs, 2001; Chew et al., 2003; Hession, 2007).

Hoplocephalus species are currently included as part of the tiger snake group with the tiger snakes (Notechis spp.) and the rough-scale snake (Tropidechis carinatus) (White, 2001; Gan et al., 2009). This inclusion is based on clinical experience and a small number of reported cases (Flecker, 1952; Comadira and Green, 1995; Fry et al., 2000; Sutherland and Tibballs, 2001; Chew et al., 2003; Hession, 2007), and because bites by Hoplocephalus spp. are often detected in the tiger snake well on the snake venom detection kit (sVDK). On this basis tiger snake antivenom (TSAV) is recommended for the treatment of Hoplocephalus bites (White, 2001), but this has not been confirmed by in vitro studies of the venoms.

The aim of this investigation was to report a series of cases of clinical envenoming by all three species of Hoplocephalus snakes. In addition, we aimed to determine the efficacy and dose of antivenom for Hoplocephalus envenoming by investigating the binding of TSAV to $H$. stephensi venom and measuring the concentrations of $H$. stephensi venom in patients' blood before and after antivenom.

\section{Materials and methods}

The study was a prospective cohort study of Hoplocephalus envenoming cases from the Australian Snakebite Project (ASP) and an in vitro examination of TSAV binding to H. stephensi venom.

\subsection{Materials}

H. stephensi (Stephen's banded snake), N. scutatus (Common tiger snake) and T. carinatus (Rough scale snake) venoms were purchased from Venom Supplies, South Australia. Each venom is pooled from multiple milkings of snakes. TSAV (Batch \# 10702) was purchased from CSL Ltd. Polyclonal monovalent rabbit IgG to $H$. stephensi venom was prepared as previously described and used in carbonate buffer (50 mM, pH 9.6) (Isbister et al., 2010a, b). Bovine serum albumin (BSA) and tetramethylbenzidine (TMB) were purchased from Sigma. PBS is phosphatebuffered saline. Rabbit IgG antibodies were biotinylated using EZ-Link Sulfo-NHS-LC-Biotin (Pierce \# 21335) and streptavidin-conjugated horseradish peroxidase was purchased from Millipore.

\subsection{Patients and patient samples}

All cases of Hoplocephalus envenoming were recruited as part of ASP. ASP is a multicentre prospective study of definite or suspected snake bites from over 120 Australian hospitals. The study design has been previously described (Isbister et al., 2008; Gan et al., 2009; Churchman et al., 2010) and approval has been obtained from all Human Research and Ethics Committees covering institutions involved.

Clinical information, laboratory testing and any treatment is recorded on datasheets for all patients recruited to ASP. This information is entered into a purpose-designed relational database (Microsoft Access $^{\mathrm{TM}}$ ). Based on the clinical and laboratory features the clinical envenoming syndrome(s) were defined in each patient based on a previously used classification (Table 1) (Ireland et al., 2010). Clinical features of any hypersensitivity reactions were recorded and graded according to Brown (2004) and a diagnosis of anaphylaxis made if a reaction met international consensus criteria (Sampson et al., 2006). Multiple serum (or plasma) samples are collected from each patient 
recruited to ASP for venom detection and quantification. Samples are spun, aliquoted, frozen and then stored at $-80{ }^{\circ} \mathrm{C}$ until assayed.

We searched the ASP database of 1017 cases of suspected or definite snakebite for the period January 2002 to March 2011 for cases of snake bite where the snake was expertly identified as a Hoplocephalus species or the snake was suspected to be a Hoplocephalus species based on geography, sVDK positive for tiger snake and patient description of the snake. In these latter cases enzyme immunoassay (EIA) was used to confirm if Hoplocephalus venom was detectable in serum samples from the patient. The snake venom detection kit (sVDK) is a commercially available qualitative enzyme immunoassay kit that can detect venom at the bite site or in urine. It gives a positive or negative to each of the venoms of the five major
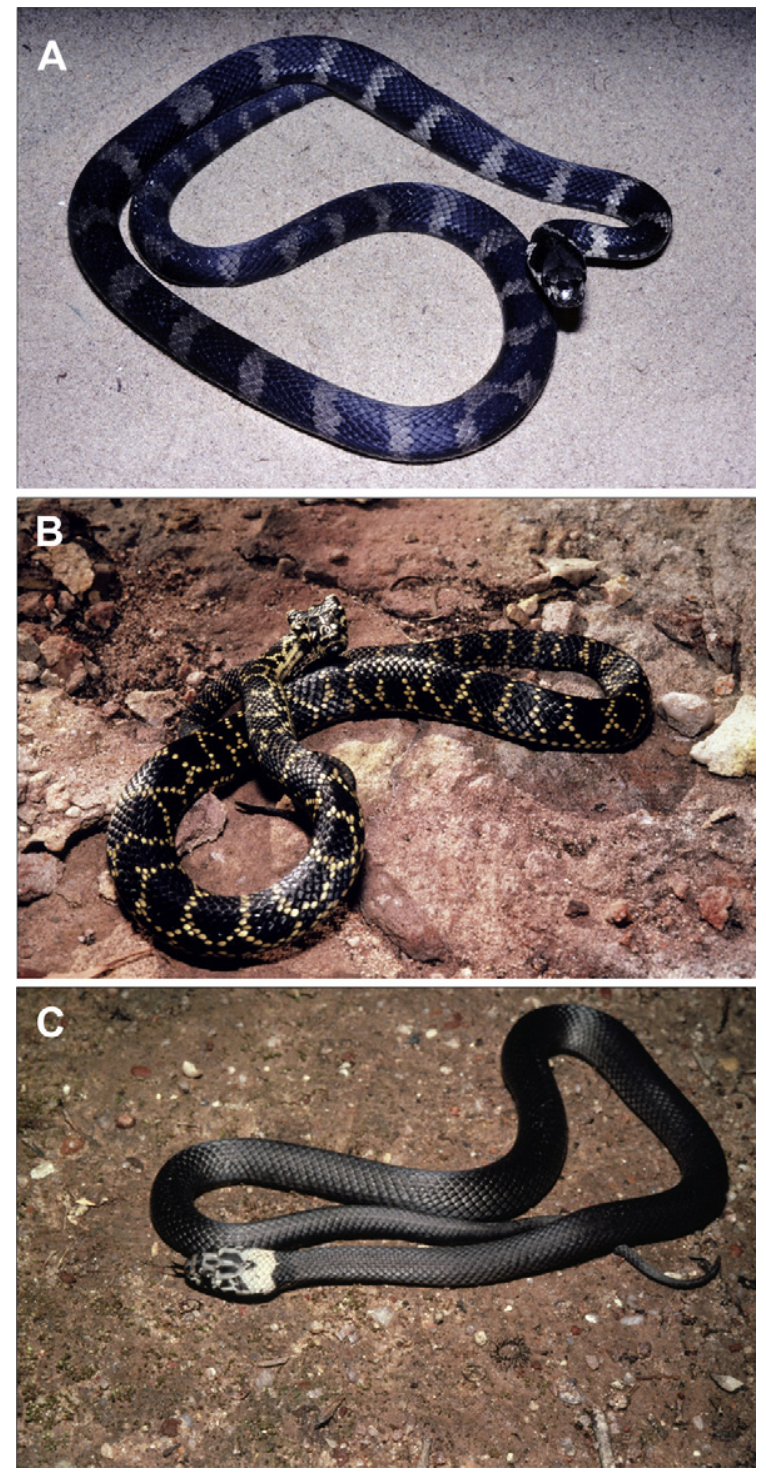

Fig. 2. Images of specimens of $H$. stephensi (A), H. bungaroides (B) and $H$. bitorquatus (C). Photographs taken by J. White.
Table 1

Classification of envenoming syndromes in snake bite cases based on clinical effects and laboratory investigations based on Ireland et al. (2010).

\begin{tabular}{ll}
\hline Envenoming syndrome & Definition \\
\hline $\begin{array}{l}\text { Venom-induced } \\
\text { consumption } \\
\text { coagulopathy } \\
\text { (VICC) } \\
\text { (Isbister et al., }\end{array}$ & \\
2010a, b) & \\
Complete & $\begin{array}{l}\text { Consumption coagulopathy with } \\
\text { undetectable fibrinogen } \\
\text { and/or a raised D-Dimer (at least } \\
\text { 10 times the assay cut-off } \\
\text { or }>2.5 \text { mg/L) and an international } \\
\text { normalised ratio (INR) }>3.0\end{array}$ \\
Incomplete consumption coagulopathy \\
with low but detectable \\
fibrinogen, elevated D-Dimer and a \\
maximum INR $<3.0$
\end{tabular}

Australian snake groups: N. scutatus (Tiger snake), Pseudonaja spp. (Brown snakes), Pseudechis spp. (Black snakes), Acanthophis spp. (Death adder) or Oxyuranus spp. (Taipan).

The following data was extracted from the ASP database for the purpose of this study: demographic information (sex, age, geographical location, circumstances of the bite), information on the snake identification (expert identification, patient description), bite site, clinical effects (including envenoming syndromes), laboratory investigations and treatment (antivenom dose and timing).

\subsection{Enzyme immunoassay (EIA)}

Enzyme immunoassays were done as previously described (O'Leary et al., 2006; Kulawickrama et al., 2010). In brief, plates were coated with rabbit anti-H. stephensi IgG $(2 \mu \mathrm{g} / \mathrm{mL})$ at room temperature, and after an hour kept overnight at $4{ }^{\circ} \mathrm{C}$. Plates were washed and blocked with $300 \mu \mathrm{L}$ of $0.5 \%$ BSA in PBS. All washes were done with PBS 
containing $0.02 \%$ TWEEN 20. After $1 \mathrm{~h}$ the plates were washed again and $100 \mu \mathrm{L}$ of human serum or plasma applied as dilutions of $0.1 \%-10 \%$ in blocking solution. After another hour the plates were washed three times and biotinylated rabbit anti-H. stephensi IgG $(100 \mu \mathrm{L}, 0.5 \mu \mathrm{g} / \mathrm{mL}$ in blocking solution) added. An hour later the plates were washed and streptavidin (100 $\mu \mathrm{L}, 1 \mu \mathrm{g} / \mathrm{mL}$ in blocking solution) added for $1 \mathrm{~h}$. Plates were washed four times, TMB $(100 \mu \mathrm{L})$ added and colour allowed to develop for 410 min until the reaction was stopped with $\mathrm{H}_{2} \mathrm{SO}_{4}(50 \mu \mathrm{L}$; $1 \mathrm{M})$. Serum samples were all measured in triplicate and average absorbance converted to a concentration from a standard curve. The limit of detection of the $H$. stephensi venom assay was $0.2 \mathrm{ng} / \mathrm{mL}$.

\subsection{In vitro antivenom binding assay}

EIA was used to measure the amount of CSL TSAV required to bind increasing concentrations of free Hoplocephalus venom in vitro, as previously described (Isbister et al., 2007). The venoms of $H$. stephensi (100 ng/mL), $N$. scutatus (100 ng/mL) and T. carinatus (100 ng/mL) in blocking solution, were mixed with increasing concentrations of TSAV $(0-100 \mathrm{mU} / \mathrm{mL})$. The venom concentrations chosen were consistent with the highest concentrations found in envenomed patients for each of the snakes (Gan et al., 2009). The venom-antivenom mixtures were pre-incubated for $1 \mathrm{~h}$ at room temperature before being applied to a plated coated with antibody corresponding to the venom (i.e. rabbit anti$H$. stephensi IgG for $H$. stephensi venom). Free venom concentration was then plotted against TSAV concentration to determine when free venom was no longer detectable.

\subsection{Data analysis}

For descriptive statistics median and interquartile ranges (IQR) were used unless the data was normally distributed. Standard curves were fitted by linear and nonlinear regression. All graphs and analyses were done in GraphPad Prism version 5.03 for Windows, GraphPad Software, San Diego California USA, www.graphpad.com.

\section{Results}

\subsection{Patient cases and venom concentrations}

There were 15 definite Hoplocephalus spp. bites including eight by Stephen's banded snakes, four by broadheaded snakes and three by pale-headed snakes. Envenoming occurred in 13 patients. The two non-envenomed patients were bitten by identified Hoplocephalus snakes. The patient was a snake handler in eight cases and the snake was in captivity in another one. The remaining six cases - four by $H$. stephensi and one by $H$. bitorquatus and one by $H$. bungaroides - occurred from non-captive snakes. The majority of cases occurred within their natural distribution despite most being kept in captivity (see Fig. 1). All of the patients were male with a median age of 33 years (IQR: 23 to 50; Range: 9-55 years). The bite was on the upper limb in 14 cases (hand [3], finger [8] and thumb [3]) and on the foot in one case.
Envenoming was similar for the three species and venom induced consumption coagulopathy (VICC) occurred in all 13 cases. An INR $>12$ occurred in seven patients. Partial VICC with only incomplete fibrinogen consumption occurred in three patients, one $H$. stephensi and two $H$. bitorquatus. The median time until the INR recovered to less than 2 was $11.5 \mathrm{~h}$ (Fig. 3). Systemic symptoms occurred in eight patients. Myotoxicity, neurotoxicity, thrombotic microangiopathy and acute renal failure did not occur. There were no cases of major haemorrhage or deaths. Local effects were minimal with local pain and bruising (Fig. 4). A comparison of the clinical features for the three species is provided in Table 2 which suggest that bites by $H$. bitorquatus are less severe with a smaller proportion with complete VICC and systemic symptoms.

Bite site sVDK was done in nine patients and was positive for tiger snake venom in eight and black snake in one (H. bitorquatus). Urine sVDK was done in one other patient and was positive for tiger snake venom. Blood was available in four of the eight $H$. stephensi bites. Venom was detected in three envenomings (1.1, 44 and $81 \mathrm{ng} / \mathrm{mL}$ ) and not detected in one $H$. stephensi bite without envenoming. In one of the three envenomings not treated with antivenom, venom was still detectable $17 \mathrm{~h}$ post-bite $(0.7 \mathrm{ng} / \mathrm{mL})$. Three $H$. bungaroides envenoming cases were tested - no venom was detected in two cases despite VICC, and $3.4 \mathrm{ng} / \mathrm{mL}$ in one with complete VICC and systemic symptoms. One $H$. bitorquatus envenoming case was tested and no venom was detected, despite the presence of VICC.

Eleven patients received antivenom, median dose 2 vials (Range: 1 to 5 vials), which was TSAV in ten cases and polyvalent antivenom in one case. No venom was detected after two vials of TSAV in the two envenomed $H$. stephensi cases treated with antivenom and no venom was detected after two vials of TSAV in the one $H$. bungaroides case where venom was detected pre-antivenom. Immediate hypersensitivity reactions occurred in six cases including one case of anaphylaxis with hypotension.

\subsection{In vitro studies}

Increasing concentrations of TSAV reduced the free venom concentration of $H$. stephensi, but not to the same

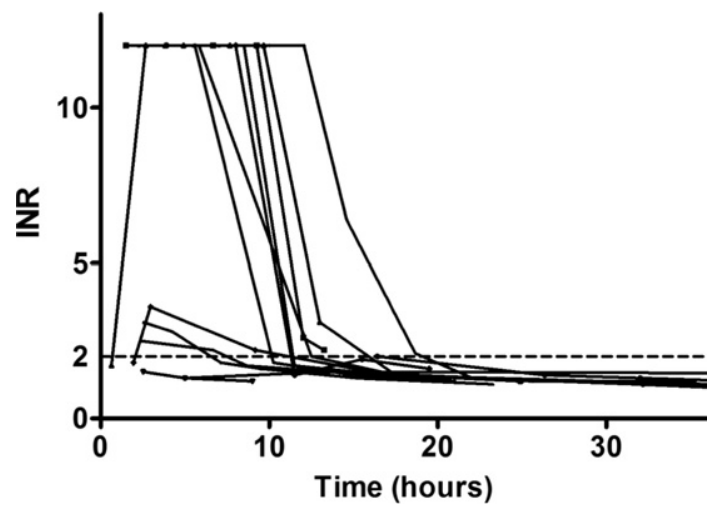

Fig. 3. Plots of the recovery of the coagulopathy comparing INR versus time for individual Hoplocephalus envenoming cases (black). Black dashed line is $\mathrm{INR}=2$. 


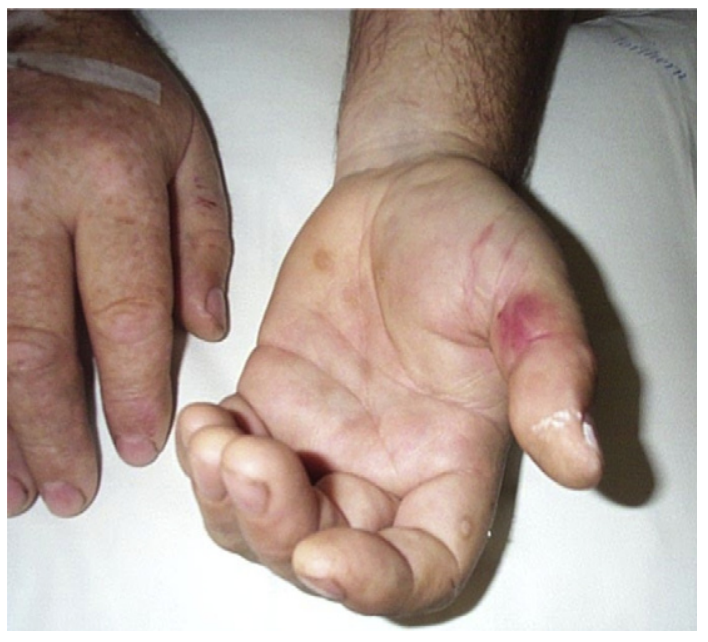

Fig. 4. Local bruising from a bite to the thumb.

degree as $N$. scutatus and T. carinatus venoms (Fig. 5). TSAV concentrations greater than $50 \mathrm{mU} / \mathrm{mL}$ reduced free $H$. stephensi venom concentrations to less than $1 \mathrm{ng} / \mathrm{mL}$ (1\%) (Fig. 5).

\section{Discussion}

In this study envenoming by all three Hoplocephalus spp. caused partial or complete VICC with no evidence of myotoxicity or neurotoxicity. Resolution of the coagulopathy was consistent with other Australasian elapids that cause VICC as shown in Fig. 3 (Isbister et al., 2009). Systemic symptoms occurred in just over half of the cases. These features of isolated VICC with only half of cases causing systemic symptoms make Hoplocephalus envenoming clinically closer to that of envenoming by Pseudonaja species (Brown snakes). However, most cases tested positive to tiger snake on the sVDK, despite a previous study indicating Holpocephalus would not be reliably detected by sVDK (Clancy et al., 1997). Of the 13 envenomed cases, 11 were treated with TSAV based on current treatment recommendations (White, 2001) and this appeared to be effective based on post-antivenom venom concentrations in a limited number of patients

Table 2

Clinical features of envenoming for each of the three species of Hoplocephalus.

\begin{tabular}{llll}
\hline Clinical syndrome & $\begin{array}{l}\text { H. stephensi } \\
N=7\end{array}$ & $\begin{array}{l}\text { H. bungaroides } \\
N=3\end{array}$ & $\begin{array}{l}\text { H. bitorquatus } \\
N=3\end{array}$ \\
\hline VICC & 7 & 3 & 3 \\
Complete & 6 & 3 & 1 \\
Partial & 1 & 0 & 2 \\
Systemic & 5 & 2 & 1 \\
$\quad$ symptoms & & & \\
Nausea & 5 & 2 & 1 \\
Vomiting & 5 & 1 & 0 \\
Headache & 2 & 2 & 2 \\
Abdominal Pain & 2 & 1 & 0 \\
Diaphoresis & 1 & 0 & 1 \\
Diarrhoea & 2 & 0 & 0 \\
\hline
\end{tabular}

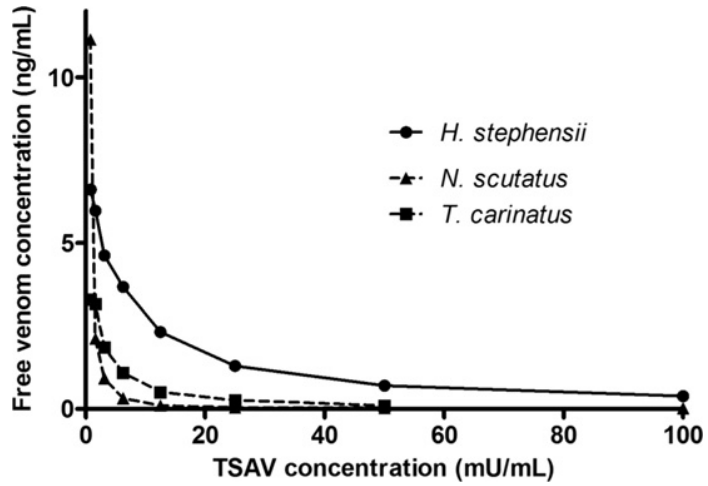

Fig. 5. The effect of increasing concentrations of TSAV on the amount of free venom detected by EIA for clinically relevant concentrations of $H$. stephensi venom (filled circle; $100 \mathrm{ng} / \mathrm{mL}$ ) compared to $N$. scutatus venom (filled square; $100 \mathrm{ng} / \mathrm{mL}$ ) and T. carinatus venom (filled triangle; $100 \mathrm{ng} / \mathrm{mL}$ ). Data are plotted as free venom concentration measured versus TSAV concentration.

and in vitro binding studies. EIA using $H$. stephensi venom did not appear to be reliable in detecting venom from the other two species.

The clinical features we describe here are similar to previous cases where VICC was reported as the predominant feature (Flecker, 1952; Comadira and Green, 1995; Fry et al., 2000; Sutherland and Tibballs, 2001; Chew et al., 2003; Hession, 2007). Flecker reported a case of envenoming by $H$. bungaroides but no laboratory studies are provided (Flecker, 1952). The patient had significant local effects but the bitten finger was cut and a ligature applied for first aid. A more recent case of $H$. bungaroides envenoming was reported in Scandinavia from a captive snake (Chew et al., 2003). This patient had complete VICC that resolved over $24 \mathrm{~h}$, minor electrocardiogram abnormalities that resolved over $8 \mathrm{~h}$ and a modest elevation in creatine kinase without clinical features of myotoxicity. They were given antivenom $11 \mathrm{~h}$ after the bite. The only other report of bites by $H$. bungaroides is in abstract form (Fry et al., 2000). Two cases were reported where VICC occurred with systemic symptoms and early collapse in one case (Personal communication, J White, 2006).

There are three previous reports of bites by $H$. stephensi (Comadira and Green, 1995; Fry et al., 2000; Hession, 2007). VICC was reported in all of these cases and the presence of systemic symptoms was consistent with our cases (Table 2). Early collapse was reported by Fry et al. (2000). There is only one previous report of envenoming by $H$. bitorquatus. (Sutherland and Tibballs, 2001) In our study envenoming by this snake appeared to be less severe, although this is based on only a small number of cases.

We have previously shown that there is little crossreactivity between $H$. stephensi venom and both $N$. scutatus and $T$. carinatus using EIA in patients with suspected $T$. carinatus envenoming (Gan et al., 2009). This was in contrast to considerable cross-reactivity between $\mathrm{N}$. scutatus and $T$. carinatus venom making it difficult to distinguish these two snakes by EIA at low venom concentrations (Gan et al., 2009). Here we demonstrate that binding of TSAV to $H$. stephensi venom is significantly less than for 
these other two snake venoms which suggests that the herpetological inclusion of Hoplocephalus within the "Tiger snake group" may be of questionable clinical relevance, despite the clear similarities between the procoagulants found in Notechis, Tropidechis and Hoplocephalus (Joseph et al., 1999; Rao et al., 2003) and the strong phylogenetic linkage (Keogh et al., 1998). Further, the clinical features of Hoplocephalus envenomings are more like Pseudonaja envenoming cases. One reason previously supporting the inclusion of Hoplocephalus with N. scutatus and T. carinatus is that many cases have been reported to be positive for tiger snake venom when the sVDK is used. Our study demonstrates that at least for $H$. bitorquatus this may not be the case.

We have previously shown that all CSL antivenoms are in fact polyvalent (O'Leary and Isbister, 2009), so it might be suggested that brown snake antivenom would be similarly effective in Hoplocephalus envenoming. One vial of brown snake antivenom or TSAV is easily sufficient to provide concentrations greater than $50 \mathrm{mU} / \mathrm{mL}$ in patients.

The in vitro binding studies demonstrate that TSAV concentrations of $50 \mathrm{mU} / \mathrm{mL}$ are sufficient to bind the majority of free $H$. stephensi venom components at concentrations above those detected in envenomed patients $(100 \mathrm{ng} / \mathrm{mL})$. The reason this occurs is likely to be the similarity of toxin groups in Australasian elapid venoms, such as the prothrombin activators, where for example there is about $98 \%$ similarity in the prothrombin activators of $H$. stephensii and T. carinatus.(Rao et al., 2003) However, Fig. 5 does indicate that the binding of TSAV to $H$. stephensi venom is not as good as $N$. scutatus venom which it is raised against or to $T$. carinatus venom which we have previously shown to be highly cross-reactivite with $N$. scutatus venom (Gan et al., 2009).

A limitation of the EIA used here is that rabbit anti-IgG was only raised against $H$. stephensi venom and we did not have antibodies to the other two Hoplocephalus venoms. Unlike previous studies using EIA to detect Pseudonaja venom (O'Leary et al., 2006; Isbister et al., 2007), where there appears to be significant cross-reactivity between species ( $P$. textilis and $P$. nuchalis), we were unable to detect venom in 2 of 3 envenomed $H$. bungaroides cases and one envenomed $H$. bitorquatus case.

\section{Conclusion}

Hoplocephalus spp. envenoming causes VICC and systemic symptoms and is clinically similar to uncomplicated brown snake (Pseudonaja spp.) envenoming. Previous studies with EIA and in vitro studies here suggest there is minimal cross reactivity between Hoplocephalus and both $N$. scutatus and T. carinatus. Within the Hoplocephalus genus itself, the venoms appear to be antigenically distinct so that our EIA, using polyclonal antibodies raised against $H$. stephensi, did not reliably detect venoms from other snakes in the genus. Hoplocephalus envenoming should be treated as a unique envenoming syndrome and not grouped with Notechis or T. carinatus. Based on in vitro studies reported here, patients may be treated with one vial of TSAV, although one vial of brown snake antivenom may also be sufficient.

\section{Ethical statement}

Approval has been obtained from all Human Research and Ethics Committees covering institutions involved for the collection of clinical information and human serum/plasma.

\section{Funding}

The study was supported in part by NHMRC Project Grant ID490305. GKI is supported by an NHMRC Clinical Career Development Award ID605817.

\section{Conflict of interest}

None declared.

\section{References}

Brown, S.G., 2004. Clinical features and severity grading of anaphylaxis. J. Allergy Clin. Immunol. 114 (2), 371-376.

Chew, M.S., Guttormsen, A.B., et al., 2003. Exotic snake bite: a challenge for the Scandinavian anesthesiologist? Acta. Anaesthesiol. Scand. 47 (2), 226-229.

Churchman, A., O'Leary, M.A., et al., 2010. Clinical effects of red-bellied black snake (Pseudechis porphyriacus) envenoming and correlation with venom concentrations: Australian Snakebite Project (ASP-11). Med. J. Aust. 193 (11-12), 696-700.

Clancy, A.M., White, J., et al., 1997. The effect of species and geographical origin of snakes on the identification of their venom using a commercial assay. Med. J. Aust. 167 (1), 56

Comadira, G.P., Green, D., 1995. Three case of envenomation by Stephen's banded snakes. Emerg. Med. Australas 7 (1), 4.

Flecker, H., 1952. Bite from broad-headed snake: Hoplocephalus bungaroides (Boie). Med. J. Aust. 1 (11), 368-369.

Fry, B.G., Masci, P.P., et al., 2000. Severe Defibrination Coagulopathy Resulting from Envenomation by the Australian Elapids Hoplocephalus Bungaroides (Broad-headed Snake) and Hoplocephalus Stephensi. (Stephen's Banded Snake) XIIIth World Congress on Animal, Plant and Microbial Toxins, Paris, IST.

Gan, M., O'Leary, M.A., et al., 2009. Envenoming by the rough-scaled snake (Tropidechis carinatus): a series of confirmed cases. Med. J. Aust. 191 (3), 183-186.

Hession, M., 2007. Stephen's Banded Snake envenomation treated with tiger snake antivenom. Emerg. Med. Australas 19 (5), 476-478.

Ireland, G., Brown, S.G., et al., 2010. Changes in serial laboratory test results in snakebite patients: when can we safely exclude envenoming? Med. J. Aust. 193 (5), 285-290.

Isbister, G.K., 2010. Snakebite doesn't cause disseminated intravascular coagulation: coagulopathy and thrombotic microangiopathy in snake envenoming. Semin. Thromb. Hemost. 36 (4), 444-451.

Isbister, G.K., O'Leary, M.A., et al., 2007. Efficacy of antivenom against the procoagulant effect of Australian brown snake (Pseudonaja sp.) venom: in vivo and in vitro studies. Toxicon 49 (1), 57-67.

Isbister, G.K., Brown, S.G., et al., 2008. Current use of Australian snake antivenoms and frequency of immediate-type hypersensitivity reactions and anaphylaxis. Med. J. Aust. 188 (8), 473-476.

Isbister, G.K., Duffull, S.B., et al., 2009. Failure of antivenom to improve recovery in Australian snakebite coagulopathy. QJM 102 (8), 563-568.

Isbister, G.K., O'Leary, M.A., et al., 2010a. Cross-neutralisation of Australian brown snake, taipan and death adder venoms by monovalent antibodies. Vaccine 28 (3), 798-802.

Isbister, G.K., Scorgie, F.E., et al., 2010b. Factor deficiencies in venominduced consumption coagulopathy resulting from Australian elapid envenomation: Australian Snakebite Project (ASP-10). J. Thromb. Haemost 8 (11), 2504-2513.

Joseph, J.S., Chung, M.C., et al., 1999. Amino acid sequence of trocarin, a prothrombin activator from Tropidechis carinatus venom: its structural similarity to coagulation factor Xa. Blood 94 (2), 621-631.

Keogh, J.S., Shine, R., et al., 1998. Phylogenetic relationships of terrestrial Australo-Papuan elapid snakes (subfamily Hydrophiinae) based on cytochrome b and 16S rRNA sequences. Mol. Phylogenet. Evol. 10 (1), 67-81.

Kulawickrama, S., O'Leary, M.A., et al., 2010. Development of a sensitive enzyme immunoassay for measuring taipan venom in serum. Toxicon 55 (8), 1510-1518. 
O'Leary, M.A., Isbister, G.K., 2009. Commercial monovalent antivenoms in Australia are polyvalent. Toxicon 54 (2), 192-195.

O’Leary, M.A., Isbister, G.K., et al., 2006. Enzyme immunoassays in brown snake (Pseudonaja spp.) envenoming: detecting venom, antivenom and venom-antivenom complexes. Toxicon 48 (1), 4-11.

Rao, V.S., Joseph, J.S., et al., 2003. Group D prothrombin activators from snake venom are structural homologues of mammalian blood coagulation factor Xa. Biochem. J. 369 (Pt 3), 635-642.
Sampson, H.A., Munoz-Furlong, A., et al., 2006. Second symposium on the definition and management of anaphylaxis: summary report-second national institute of allergy and infectious disease/food allergy and anaphylaxis network symposium. J. Allergy Clin. Immunol. 117 (2), 391-397.

Sutherland, S.K., Tibballs, J., 2001. Genus Hoplocephalus. In: Sutherland, S. K., Tibballs, J. (Eds.), Australian Animal Toxins, vol. 2. Oxford University Press, Melbourne, pp. 209-219.

White, J., 2001. CSL Antivenom Handbook. CSL Ltd, Melbourne. 